\title{
Localization of the primary sites of involvement in the spinal sensory and motor pathways for multilevel MRI abnormalities in degenerative cervical myelopathy
}

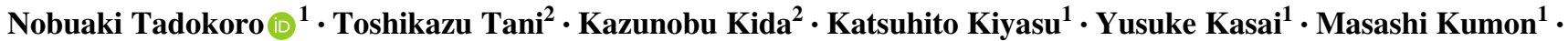 \\ Ryuichi Takemasa $^{1} \cdot$ Masahiko Ikeuchi $^{1}$
}

Received: 12 July 2017 / Revised: 23 August 2017 / Accepted: 24 August 2017 / Published online: 30 October 2017

(C) International Spinal Cord Society 2018

\begin{abstract}
Study design Exploratory clinical study.

Objectives To localize the sites of conduction block in the spinal sensory and motor pathways for minimizing the level of surgical intervention despite MRI evidence of compression at several levels in degenerative cervical myelopathy (DCM).
\end{abstract}

Setting Kochi Medical School Hospital, Japan.

Methods We analyzed 83 DCM patients (69 \pm 12 years) who underwent serial intervertebral recording of both ascending (A-SCEPs) and descending spinal cord-evoked potentials (D-SCEPs) after epidural and transcranial stimulation, respectively, during surgery before decompression procedures. The site of conduction block was identified by an abrupt reduction of the negative peak accompanied by an enlargement of the initial-positive peak. T1-weighted MRI allowed quantitative assessments of cord compression in relation to the level of conduction block.

Results The A-SCEP and D-SCEP studies revealed conduction blocks at the same single level in 78 patients (94\%) and at two separate levels in five patients $(6 \%)$ for MRI abnormalities extending to $3.1 \pm 1.1$ levels. The site of conduction block had intense cord compression on MRI with either the smallest (81\%) or the second smallest (19\%) anteroposterior diameter and cross-sectional area of the cord. Based on the SCEP findings, 44 patients were treated by a single-level (35) or a two-level (9) anterior operation for MRI abnormalities of $2.7 \pm 1.1$ levels.

Conclusions Combined A-SCEP and D-SCEP studies served as useful additions to MRI in localizing the primary sites responsible for myelopathy, which helped exclude clinically silent compressions to minimize surgical intervention in 34 patients with anterior operation.

\section{Introduction}

Non-traumatic, degenerative forms of cervical myelopathy termed "degenerative cervical myelopathy" (DCM) [1] represent the commonest form of spinal cord disorder in the elderly. The selections of operative approach and the

Nobuaki Tadokoro

tadokoro.nobuaki@kochi-u.ac.jp

1 Department of Orthopaedic Surgery, Kochi Medical School, Kochi University, Kohasu Oko-chou, Nankoku 783-8505, Japan

2 Department of Orthopaedic Surgery, Kubokawa Hospital, Shimanto, Japan vertebral levels to be surgically decompressed for DCM generally rely heavily on morphological changes shown by MRI. However, as described previously, MRI tends to show clinically silent compression at an increasingly greater number of levels with advancing age [2].

Our previous electrophysiological studies in DCM indicated that ascending spinal cord-evoked potentials (A-SCEPs) recorded intraoperatively at multiple sites along the spinal cord after epidural stimulation caudally can identify the site of conduction block, which, among the physiologic consequences of spinal cord compression, probably plays the most important part in producing potentially treatable clinical deficits [3, 4], and thereby guiding the surgeon to the most appropriate level for 
Table 1 Functional scoring system for cervical myelopathy (adapted from Japanese orthopaedic association [6])

\begin{tabular}{lll}
\hline Function & Score & Remarks \\
\hline Motor function of upper limbs & 0 & Unable to feed oneself \\
& 1 & Unable to handle chopsticks but able to eat with a \\
spoon \\
& 2 & Handle chopsticks with much difficulty \\
& 3 & Handle chopsticks with slight difficulty \\
& 4 & Normal finger dexterity \\
Motor function of lower limbs & 0 & Chairbound \\
& 1 & Requires walking aids \\
& 2 & Needs support when going up and down stairs \\
Sensory function of upper limbs, lower limbs, & 3 & Capable of fast walking but with some difficulty \\
and trunk & 0 & Normal walking ability \\
& 4 & Severe sensory loss \\
Sphincter function & 1 & Mild sensory loss \\
& 2 & Normal \\
& 0 & Retention and/or incontinence \\
& 1 & Sense of retention and/or incomplete continence \\
& 2 & Retardation and/or pollakiuria \\
& 3 & Normal \\
\hline
\end{tabular}

surgical intervention. This electrophysiological technique, however, has the inherent limitation of only detecting the most caudal conduction block, which precludes the evaluation of more rostral segments. An additional assessment of descending spinal cord-evoked potentials (D-SCEPs) after transcranial electrical stimulation of the brain circumvents this problem, uncovering the most rostral conduction block [5].

In the present study, we analyzed a series of 83 DCM patients who underwent both A-SCEP and D-SCEP studies during surgery, before decompression procedures, to investigate a correlation between electrophysiological and MRI abnormalities.

\section{Methods}

\section{Patients}

From January 2004 to March 2013, 98 DCM patients with moderate to severe spastic limb paresis underwent intraoperative assessments of both A-SCEP and D-SCEP. Excluding 15 patients with a history of previous cervical spine surgery (14) or trauma causing cord damage immediately after injury (1), we analyzed a total of 83 patients (47 men and 36 women) aged $69 \pm 12$ (mean \pm SD) years. None had a history of seizures or medical implants, potentially incompatible with safe transcranial electrical stimulation and MRI examinations, respectively. All agreed in writing to participate in the study after reading and signing an informed consent form approved by the hospital ethics committee.

Myelopathy resulted from cervical spondylosis in 61 patients, ossification of the posterior longitudinal ligament in 13, rheumatoid arthritis in 6, destructive spondyloarthropathy in 2 , and calcification of the ligamentum flavum in 1 . The surgical procedures for 83 patients comprised an anterior operation in 44 patients including a single-level anterior operation in 35 , a posterior operation in 38 and combined anterior and posterior operations in 1.

\section{Clinical findings}

For functional assessment of DCM patients, we used the functional scale developed by the Japanese Orthopaedic Association (JOA) [6], which scores motor function of the upper and lower limbs from 0 to 4 points each, bladder function from 0 to 3 points, and sensory function of the upper limbs, lower limbs, and trunk from 0 to 2 points each for the total score ranging from 0 to 17 points (Table 1). The JOA scores of 83 patients averaged $1.7 \pm 1.1$ for motor function of the upper limbs, $1.4 \pm 0.9$ for motor function of the lower limbs, $1.9 \pm 1.0$ for bladder function, and $3.5 \pm$ 1.3 for sensory function. The total JOA scores averaged 8.4 \pm 3.0 , ranging from 2 to 14 . Although muscle stretch reflexes were generally hyperactive, responses were diminished for the biceps in 3 patients, for the triceps in 4 , and for the gastrocnemius-soleus in 18. Extensor plantar responses were elicited in 33 patients. 


\section{Electrodiagnosis}

\section{Stimulation}

We conducted all SCEP studies in the operating room of Kochi Medical School Hospital after preoperative general anesthesia with sevoflurane or propofol. For evoking the ASCEPs, we used a pair of stimulating electrodes (NM-212B, Nihon Koden, Tokyo, Japan), with two platinum tips at the end of an 18-gauge polyethylene tube, percutaneously introduced into dorsal epidural space at the lumbar or lower thoracic level via a Tuohy needle. Electrical stimulation consisted of a square wave, $0.1 \mathrm{~ms}$ in duration and of $20-40 \mathrm{~mA}$ in intensity, delivered at a rate of 3-20/s.

For evoking the D-SCEPs, we placed self-securing lower impedance spiral corkscrew-type needles (CS001-220, AGram, Glenn Rock, New Jersey) into the scalp $2 \mathrm{~cm}$ anteriorly and $5 \mathrm{~cm}$ laterally to the vertex on both sides [5]. With this stimulation montage, a high-voltage electrical stimulator (Digitimer D185, Welwyn Garden City, UK) delivered a transcranial electrical stimulation pulse of $50 \mu \mathrm{s}$ in duration and up to $400 \mathrm{~V}$ in intensity at a rate of $1 / \mathrm{s}$, stimulating right and left motor cortexes alternately with reversed electrode polarity.

\section{Recording}

In an anterior operation, we inserted a series of monopolar needle electrodes, $0.7 \mathrm{~mm}$ in diameter and about $4 \mathrm{k} \Omega$ in impedance at $1 \mathrm{kHz}$ (OA210-006, Unique Medical Corp, Tokyo, Japan), into the intervertebral discs in the midline after exposure of the anterior aspect of the vertebral bodies $[3,4]$. They were then advanced posteriorly within the distance of the disc diameter measured on the plain lateral radiograph and calculated with magnification factor to avoid penetrating the dura. In a posterior operation, we inserted the same needle electrodes as used in anterior operations into the ligamentum flavum in the midline at serial intervertebral spaces after exposure of the posterior aspect of the vertebrae [4]. A needle electrode, inserted into the skin at the rostral end of the operative field for A-SCEPs and at its caudal end for D-SCEPs, served as the common reference [5]. A pair of alligator clips was attached to the skin at the operative site as the ground electrode. A muscle relaxant (vecuronium, $\quad 0.015-0.110 \mathrm{mg} / \mathrm{kg}$ bolus), intravenously administered immediately before recording SCEPs, abolished interference from twitching paraspinal muscles near the recording electrodes. Each test set comprised an average of 200 and 50 summated potentials for A-SCEPs and DSCEPs, respectively, sampled at $50 \mathrm{kHz}$, with a frequency response of $20 \mathrm{~Hz}-3 \mathrm{kHz}$. An eight-channel averager (Neuropak MEB2200, Nihon Kohden, Tokyo, Japan)

\section{D-SCEPs}

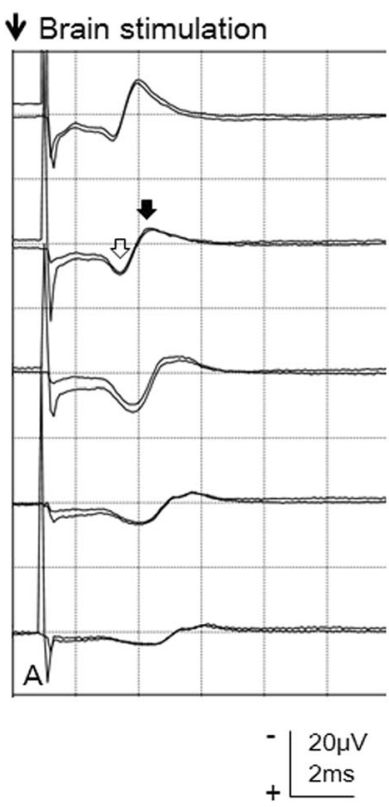

A-SCEPs

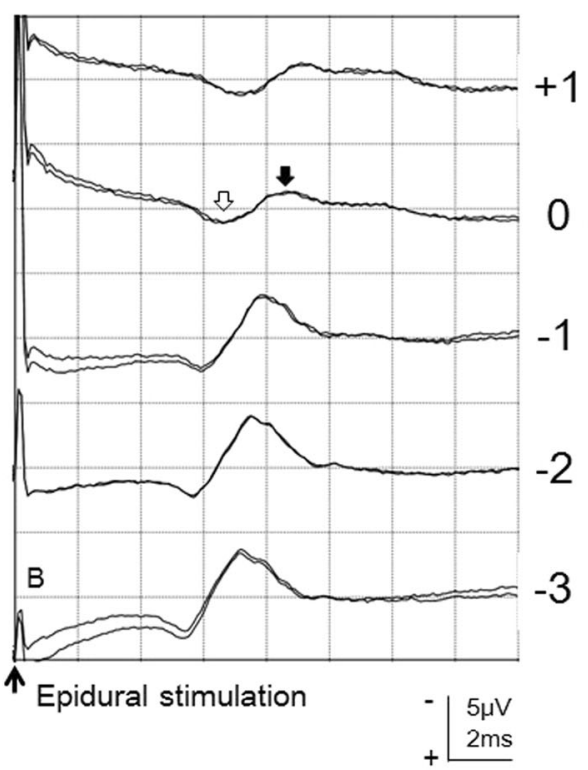

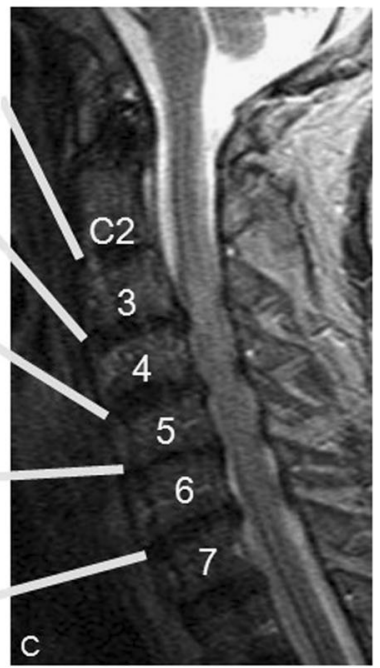

Fig. 1 Anterior recording of D-SCEPs (a) and A-SCEPs (b), and a sagittal T2-weighted MRI (c) in a 75-year-old woman with cervical spondylotic myelopathy. Both D-SCEPs and A-SCEPs demonstrate abrupt reduction of the negative peaks (solid arrows) with concomitant augmentation of the initial-positive peaks (open arrows), indicating conduction blocks at $\mathrm{C} 3-4$ in the motor and sensory pathways. With the help of SCEP studies, the patient was successfully treated by a single-level anterior operation at $\mathrm{C} 3-4$ in the presence of "MRI abnormalities" at C3-4 through C5-6 
Fig. 2 Anterior recording of DSCEPs (a) and A-SCEPs (b) and a sagittal T2-weighted MRI (c) in an 80-year-old man with cervical spondylotic myelopathy. Both D-SCEPs and A-SCEPs demonstrate abrupt reduction of the negative peaks (solid arrows) with concomitant augmentation of the initialpositive peaks (open arrows) at C3-4, indicating conduction blocks at $\mathrm{C} 3-4$ in the motor and sensory pathways. With the help of SCEP studies, the patient was successfully treated by a singlelevel anterior operation at C3-4 in the presence of "MRI abnormalities" at $\mathrm{C} 2-3$ through C5-6

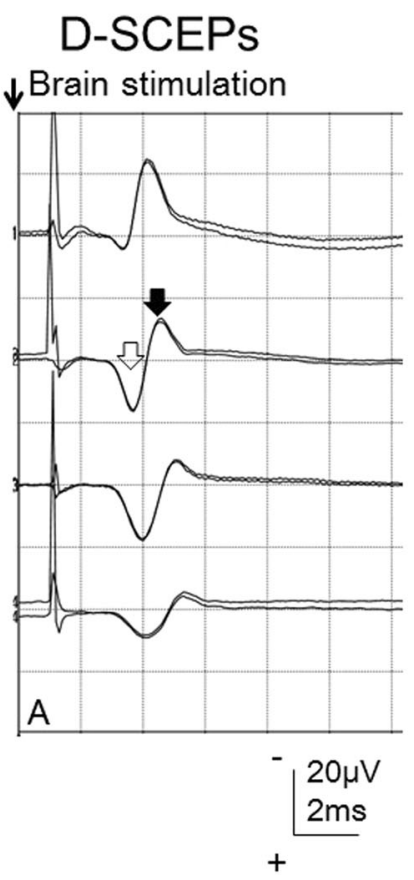

\section{A-SCEPS}

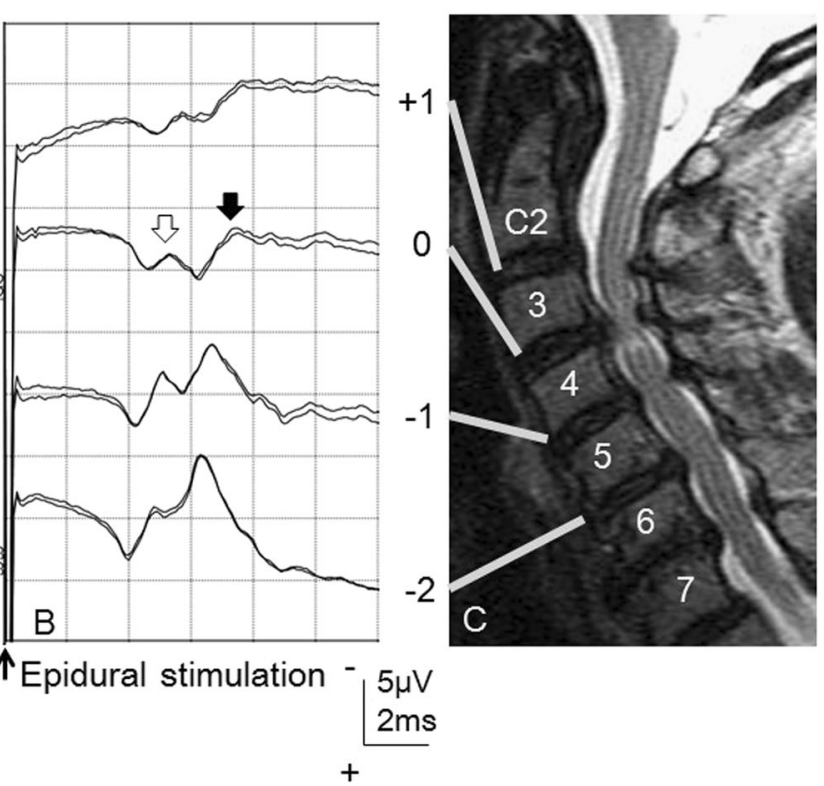

myelogram effect", clearly depicting the subarachnoid space obstruction by compression. In the current study, we used the term "MRI abnormalities" defined as an increased T2 signal within the spinal cord or compression-induced subarachnoid space obstruction on mid-sagittal T2-weighted MRIs or both.

\section{Statistical analysis} conduction block identified by abrupt reduction in amplitude of the SCEP negative peak, with the other levels numbered in order of increasing distance from the " 0 " level, assigning a minus sign caudally. Measurements of SCEPs included: (1) latencies from the stimulus artifacts to the initial-positive peaks, (2) amplitudes from the baseline to the initial-positive and the negative peaks, and (3) area (voltage-time integral) of the initial-positive and the negative peaks.

\section{MRI evaluation}

All patients underwent surface coil MR examination of cervical cord preoperatively with the superconducting system (1.5 T Signa HDx; GE Healthcare, Waukesha, WI, USA). The spin echo pulse sequences were 350-600/9-12 (TR ms/TE ms) for T1-weighted images and 2600-4000/90110 for T2-weighted images. Cord measurements at each intervertebral level from $\mathrm{C} 2-3$ to C6-7 included: (1) anteroposterior diameter on midsagittal $\mathrm{T} 1$-weighted images and (2) cross-sectional area on axial T1-weighted images. T2-weighted images served best to detect increased signal intensity resulting from cord compression. These long TR/ TE MRI techniques also produce a so-called "pseudo
We used Wilcoxon's signed-rank test for evaluating paired data and one-way ANOVA followed by Tukey HSD test for unpaired data. Values were given as the mean and with a 95\% confidence interval. Two-tailed tests were considered significant when $p<0.05$. We used JMP statistical software (Version 12.01, SAS Institute, Cary, NC) for all statistical analysis.

\section{Statement of ethics}

We certify that all applicable institutional and governmental regulations concerning the ethical use of human volunteers were followed in the course of this research.

\section{Results}

\section{A-SCEPs}

Segmental A-SCEP studies in short increments identified the site of focal conduction block by showing an abrupt reduction in size of the negative peak accompanied by an 
Fig. 3 Anterior recording of DSCEPs (a) and A-SCEPs (b) and a sagittal T2-weighted MRI (c) in a 60-year-old woman with cervical spondylotic myelopathy. The D-SCEPs and A-SCEPs demonstrate abrupt reduction of the negative peaks (solid arrows) with concomitant augmentation of the initialpositive peaks (open arrows) at C5-6 and C7-T1, respectively, indicating a conduction block at C5-6 in the motor pathways and at $\mathrm{C} 7-\mathrm{T} 1$ in the sensory pathways. With the help of SCEP studies, the patient was successfully treated by a twolevel anterior operation at C5-6 and $\mathrm{C} 7-\mathrm{T} 1$ in the presence of 'MRI abnormalities' at C5-6 through T1-2
D-SCEPS

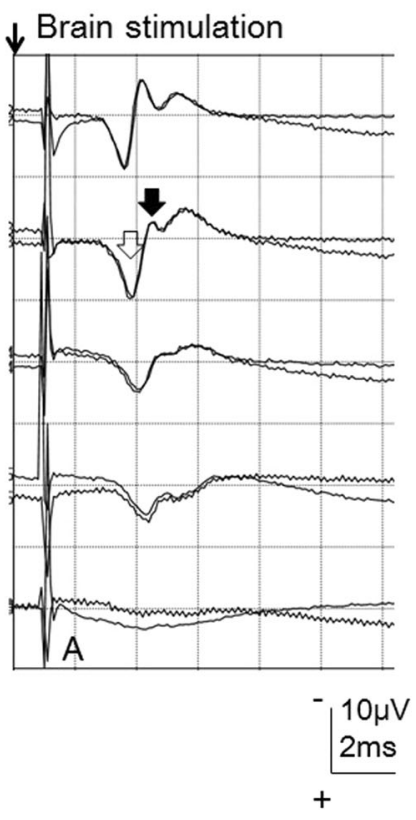

A-SCEPS
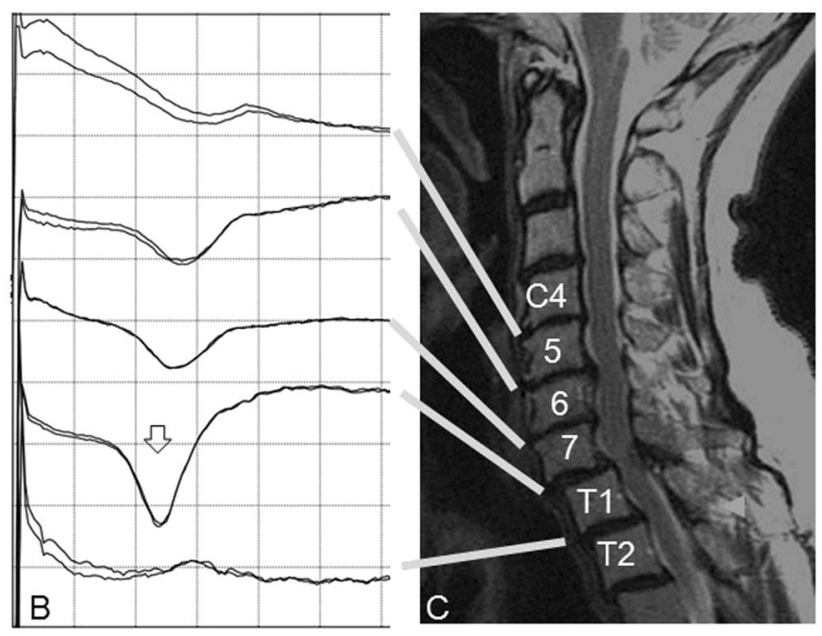

$\uparrow$ Epidural stimulation

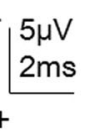

Table 2 Negative and initial-positive components of A-SCEP

\begin{tabular}{|c|c|c|c|c|c|c|c|c|c|}
\hline \multirow{3}{*}{$\begin{array}{l}\text { Recording } \\
\text { level }\end{array}$} & \multirow{3}{*}{$\begin{array}{l}\text { Number of } \\
\text { patients }\end{array}$} & \multicolumn{4}{|c|}{ Negative component } & \multicolumn{4}{|c|}{ Initial-positive component } \\
\hline & & $\begin{array}{l}\text { Amplitude } \\
(\%)\end{array}$ & $p$-value* & Area $(\%)$ & $p$-value* & $\begin{array}{l}\text { Amplitude } \\
(\%)\end{array}$ & $p$-value* & Area $(\%)$ & $p$-value* \\
\hline & & $\begin{array}{l}\text { Mean }(95 \% \\
\text { CI) }\end{array}$ & & $\begin{array}{l}\text { Mean }(95 \% \\
\text { CI) }\end{array}$ & & $\begin{array}{l}\text { Mean }(95 \% \\
\text { CI) }\end{array}$ & & $\begin{array}{l}\text { Mean }(95 \% \\
\text { CI) }\end{array}$ & \\
\hline \multirow[t]{2}{*}{2} & 28 & $25(17-33)$ & & $25(15-35)$ & & $115(71-159)$ & & $224(71-376)$ & \\
\hline & & & $p<0.0001$ & & $p<0.0001$ & & $p<0.0001$ & & $p<0.0001$ \\
\hline \multirow[t]{2}{*}{1} & 83 & $28(21-34)$ & & $25(18-31)$ & & $\begin{array}{l}152 \\
(128-176)\end{array}$ & & $\begin{array}{l}314 \\
(229-400)\end{array}$ & \\
\hline & & & $p<0.0001$ & & $p<0.0001$ & & $p=0.04$ & & NS \\
\hline \multirow[t]{2}{*}{0} & 83 & $38(32-44)$ & & $36(29-42)$ & & $\begin{array}{l}170 \\
(133-206)\end{array}$ & & $\begin{array}{l}288 \\
(206-370)\end{array}$ & \\
\hline & & & $p<0.0001$ & & $p<0.0001$ & & $p<0.0001$ & & $p<0.0001$ \\
\hline \multirow[t]{2}{*}{-1} & 83 & $100(100)$ & & $100(100)$ & & $100(100)$ & & $100(100)$ & \\
\hline & & & NS & & NS & & NS & & NS \\
\hline \multirow[t]{2}{*}{-2} & 64 & $106(95-117)$ & & $\begin{array}{l}114 \\
(95-132)\end{array}$ & & $\begin{array}{l}111 \\
(102-121)\end{array}$ & & $110(91-130)$ & \\
\hline & & & NS & & NS & & NS & & NS \\
\hline-3 & 25 & $100(82-117)$ & & $92(70-114)$ & & $113(90-136)$ & & $115(79-150)$ & \\
\hline
\end{tabular}

"0" represents the site of conduction block, with the other levels numbered in order of increasing distance from the "0" level, assigning a minus sign caudally

CI confidence interval

*Calculated according to Wilcoxon's signed-rank test

enlargement of the initial positive peak in all patients (Figs. 1-3). At this level, designated as " 0 ", the negative peaks significantly reduced in amplitude (38 (32-44)\%; $p<0.0001)$ and area $(36(29-42) \% ; p<0.0001)$ compared to the " -1 " level $(100 \%)$, which was taken as the baseline
(Table 2). In contrast, the initial-positive peak increased in amplitude (170 (133-206)\%; $p<0.0001)$, and area (288 (206-370)\%; $p<0.0001$ ) (Table 2). In addition, the latency increase was greater from " -1 " to "0" $(0.7(0.6-0.8) \mathrm{ms})$ 
Table 3 Negative and initial-positive components of D-SCEP

\begin{tabular}{|c|c|c|c|c|c|c|c|c|c|}
\hline \multirow[t]{3}{*}{ Recording level } & \multirow[t]{3}{*}{ Number of patients } & \multicolumn{4}{|c|}{ Negative component } & \multicolumn{4}{|c|}{ Initial-positive component } \\
\hline & & Amplitude (\%) & $p$-value* & Area $(\%)$ & $p$-value* & Amplitude (\%) & $p$-value* & Area $(\%)$ & $p$-value* \\
\hline & & Mean $(95 \% \mathrm{CI})$ & & Mean $(95 \%$ CI $)$ & & Mean $(95 \% \mathrm{CI})$ & & Mean $(95 \% \mathrm{CI})$ & \\
\hline \multirow[t]{2}{*}{2} & 27 & $107(92-121)$ & & $107(88-126)$ & & $88(76-101)$ & & $88(65-111)$ & \\
\hline & & & NS & & NS & & NS & & NS \\
\hline \multirow[t]{2}{*}{1} & 83 & $100(100)$ & & $100(100)$ & & $100(100)$ & & $100(100)$ & \\
\hline & & & $p<0.0001$ & & $p<0.0001$ & & $p<0.0001$ & & $p<0.0001$ \\
\hline \multirow[t]{2}{*}{0} & 83 & $46(38-53)$ & & $44(36-52)$ & & $149(130-168)$ & & $260(191-329)$ & \\
\hline & & & $p<0.0001$ & & $p<0.0001$ & & $p<0.0001$ & & NS \\
\hline \multirow[t]{2}{*}{-1} & 83 & $27(18-35)$ & & $22(16-29)$ & & $125(102-147)$ & & $313(221-406)$ & \\
\hline & & & $p<0.0001$ & & $p<0.0001$ & & $p<0.0001$ & & $p<0.0001$ \\
\hline \multirow[t]{2}{*}{-2} & 64 & $16(11-21)$ & & $15(10-20)$ & & $78(63-93)$ & & $224(138-310)$ & \\
\hline & & & $p=0.01$ & & $p=0.002$ & & $p<0.0001$ & & $p<0.0001$ \\
\hline-3 & 27 & $14(6-21)$ & & $12(6-18)$ & & $56(44-66)$ & & $128(74-181)$ & \\
\hline
\end{tabular}

" 0 " represents the site of conduction block, with the other levels numbered in order of increasing distance from the "0" level, assigning a minus sign caudally

CI confidence interval

*Calculated according to Wilcoxon's signed-rank test

compared to the value from " -2 " to " -1 " $(0.4(0.3-0.5) \mathrm{ms})$ $(p<0.0001)$.

\section{D-SCEPs}

Similarly, incremental D-SCEP studies disclosed a single site of focal conduction block in all patients (Figs. 1-3). At this level ("0"), the negative peaks significantly reduced in amplitude (46 (38-53)\%; $p<0.0001)$, and area (44 $(36-52) \% ; p<0.0001)$ compared to the baseline at " +1 " level $(100 \%)$ (Table 3). In contrast, the initial-positive peak significantly increased in amplitude (149 (130-168)\%; $p<$ $0.0001)$, and area (260 (191-329)\%; $p<0.0001)$ (Table 3).

\section{A-SCEPs vs D-SCEPs}

Both A-SCEP and D-SCEP studies uncovered a single site of conduction block at the same level in 78 out of 83 patients (94\%), 36 at C3-4 (Figs. 1 and 2), 28 at C4-5, 10 at C5-6, and 4 at C6-7. In the remaining five patients $(6 \%)$, the two studies revealed conduction block at two separate levels; A-SCEPs and D-SCEPs, respectively, showing the conduction block at $\mathrm{C} 4-5$ and $\mathrm{C} 3-4$ in two patients, at C5-6 and C4-5, at C6-7 and C5-6 in one patient each, and at $\mathrm{C} 7-\mathrm{T} 1$ and $\mathrm{C} 5-6$ in one patient (Fig. 3).

\section{MRI}

Of the five intervertebral levels from $\mathrm{C} 2-3$ to $\mathrm{C} 6-7$ assessed by mid-sagittal T2-weighted MRI, the number of intervertebral levels of "MRI abnormalities" averaged 3.1 \pm 1.1 (range, 1-5) for the total 83 patients. T2-weighted MRI abnormalities consisted of the subarachnoid space obstructions at a total of 261 intervertebral levels (anteriorly at 46, posteriorly at 37 or both anteriorly and posteriorly at 178) and high-intensity spinal cord signals extending to a total of 103 intervertebral levels in 79 out of 83 patients. Eighty-one out of one-hundred three such high-signal intervertebral levels (79\%) matched the site of conduction block ("0") and the remaining $22(21 \%)$ were located at " -1 " (8), at " +1 " (6), at " -2 " (7), or at " +2 " (1).

With T1-weighted MRI, an overall quantitative assessment of cord compression in relation to the level of conduction block ("0") showed that the "0" level had a significantly smaller anteroposterior diameter (4.2 (3.9-4.4) $\mathrm{mm} ; p<0.0001)$ and cross-sectional area of the cord (61 (58-63) $\left.\mathrm{mm}^{2} ; p<0.0001\right)$ than the remaining more caudal or rostral levels (Table 4). For the 78 patients in whom ASCEPs and D-SCEPs uncovered conduction block at the same single levels, these levels each corresponded to those with the smallest anteroposterior diameter and crosssectional area of the cord in 66 patients $(85 \%)$, and to those with the second smallest anteroposterior diameter and cross-sectional area of the cord in 12 patients $(15 \%)$. The latter group of patients showing the second smallest, and not the smallest, anteroposterior diameter and crosssectional area of the cord at the "0" levels tended to have maximal cord involvement at the cervical enlargement (i.e., at C5-6 or C6-7).

In the five patients in whom A-SCEPs and D-SCEPs disclosed conduction block at two separate levels, those levels had either the smallest or the second smallest anteroposterior diameter and cross-sectional area of the cord. A rare cord involvement at $\mathrm{C} 7-\mathrm{T} 1$, detected by the A-SCEP 
Table 4 Cervical cord measurement in relation to the level of conduction block (0 level)

\begin{tabular}{llll}
\hline $\begin{array}{l}\text { Intervertebral } \\
\text { level }\end{array}$ & $\begin{array}{l}\text { Number of } \\
\text { patients }\end{array}$ & $\begin{array}{l}\text { Anteroposterior } \\
\text { diameter }(\mathrm{mm}) \text { mean } \\
(95 \% \mathrm{CI})\end{array}$ & $\begin{array}{l}\text { Cross- } \\
\text { sectional area } \\
\left(\mathrm{mm}^{2}\right) \text { mean } \\
(95 \% \mathrm{CI})\end{array}$ \\
\hline 2 & 47 & $6.4(6.1-6.7)$ & $78(74-82)$ \\
1 & 83 & $6.1(5.9-6.4)$ & $77(74-79)$ \\
0 & 83 & $4.2(3.9-4.4)^{*}$ & $61(58-63)^{* *}$ \\
-1 & 83 & $5.8(5.5-6.0)$ & $72(69-75)$ \\
-2 & 65 & $5.8(5.6-5.9)$ & $71(68-74)$ \\
-3 & 36 & $5.9(5.6-6.1)$ & $70(67-73)$ \\
\hline
\end{tabular}

One-way ANOVA followed by Tukey HSD test is used for statistical analysis

" 0 " represents the site of conduction block, with the other levels numbered in order of increasing distance from the " 0 " level, assigning a minus sign caudally

Anteroposterior diameter of the cord was measured on midsagittal T1weighted MRI

Cross-sectional area of the cord was measured on axial T1-weighted MRI

CI confidence interval

*Significantly smaller $(p<0.0001)$ compared to more rostral and caudal levels

**Significantly smaller compared to more rostral and caudal levels with $p<0.0001$ except for " -3 " level with $p=0.004$

study in one patient, resulted from MRI evidence of cord compression due to $\mathrm{C} 7$ degenerative spondylolisthesis (Fig. 3).

\section{Influence of SCEP findings on length of fusion decisions}

Laminoplasty was applied at multiple levels in all 39 patients treated by posterior operation for addressing their developmental stenosis, even though they had a single or a two-level SCEP abnormality. In contrast, all 44 patients treated by anterior operation underwent anterior decompression followed by fusion at either a single level (35) or a two level (9) based on the SCEP findings, in spite of MRI abnormalities extending to $2.7 \pm 1.1$ levels. In particular, the SCEP studies helped reduce the surgical intervention to a single appropriate level for MRI abnormalities of $3.0 \pm$ 0.8 levels (range, 2-4 levels) in 29 patients and reduce to two levels for MRI abnormalities of $3.8 \pm 0.8$ levels (range, 3-5 levels) in five patients with progressive functional recovery after operation.

\section{Discussion}

In compression neuropathies, conduction block, a failure of impulse propagation across the lesion site, results from compression-induced segmental demyelination [7], focal nerve ischemia [8] or both, causing reversible, and therefore treatable, clinical weakness and sensory loss. Similarly, in compression myelopathies, conduction block probably plays an important role in producing potentially treatable clinical deficits [9]. We reported previously that segmental A-SCEP studies in short increments can precisely localize the site of conduction block [3, 4]. At this site, the SCEPs showed the combination of the two opposite changes, an abrupt reduction in size of the negative peak accompanied by an enlargement of the initial positive peak, which helps to document the site of abnormality and may otherwise escape detection.

This principle of waveform changes holds for analyses of both A-SCEP [3, 4] and D-SCEP [5] in identifying focal conduction block. In short, at the site of conduction block, a blocked fiber contributes to a normal positivity followed by a substantially reduced negativity, as the impulse approaches without reaching the recording site. This reduction in negativity not only decreases the negative peak of the SCEP but also increases its positive peak resulting from loss of physiologic phase cancellation [9]. At the sites beyond the block, a blocked fiber gives rise to a killed-end effect with a volume conducted positive wave (Fig. 3) [3-5].

The A-SCEPs mainly represent sensory responses mediated by the dorsal spinocerebellar tract and the dorsal columns as evidenced in previous studies [10], whereas the D-SCEPs consist of the so-called "direct" or D-waves produced by excitation of the corticospinal tract axons by transcranial electrical stimulation [5, 10, 11]. Thus, the present study showed that both sensory and motor pathways are usually, though not always, functionally compromised at the same single level $(94 \%$ in this series of DCM patients).

The site of conduction block identified by the A-SCEP and the D-SCEP, whether the two studies revealed conduction block at the same single level (78 out of 83 patients) or at two separate levels (5 out of 83 patients), corresponded to the intervertebral level of sufficient degree of cord compression with MRI evidence of either the smallest anteroposterior diameter and cross-sectional area of the cord (for $81 \%$ of 88 conduction blocks) or the second smallest anteroposterior diameter and crosssectional area of the cord (for $19 \%$ of 88 conduction blocks). This finding indicates the functional importance of direct mechanical pressure on the white matter rather than vascular factors involving the gray matter remote from the site of compression, thus causing false localizing signs [12]. Measuring the absolute values of anteroposterior diameter and cross-sectional area of the cord has a limitation in estimating the degree of cord compression, because the spinal cord varies in size depending on the level; the anteroposterior diameter normally decreases, and the 
cross-sectional area increases progressively toward the lower cervical level [13]. Therefore, some patients, who had the second smallest cross-sectional area of the cord at the cervical enlargement with conduction block, may have in effect the most intense cord compression at that level. The site of conduction block also corresponded to the level of high-intensity spinal cord signals on T2-weighted MRIs, which, however, were located more extensively, spreading to the neighboring regions or located at separate levels. The high-intensity signals without conduction block at 22 out of 103 high-signal sites (21\%) may have resulted from pathological changes in the cord segments confined to the gray matter [14].

As we have shown previously with a single-modality study of A-SCEP in a series of 129 patients of cervical spondylotic myelopathy representing all age groups, the primary sites of cord involvement shift from lower to upper cervical levels with aging, even though age-related degenerative radiological changes of the cervical spine abound at lower cervical levels [4]. The present data derived from a relatively older cohort of DCM patients, with an average age of 69 years, confirmed a high incidence of conduction blocks at C3-4 or C4-5 not only in spinal sensory pathways $(80 \%)$ but also in motor pathways $(81 \%)$.

Both A-SCEPs and D-SCEPs originate from axonal volleys of the spinal tracts without intervening synapses, providing a good quality measure with relatively large, synchronous, anesthesia-resistant responses for rapid feedback to the surgeons. Precise localization of the primary sites of involvement in the spinal sensory and motor pathways plays a particularly important role in appropriately minimizing the level of anterior surgical intervention in the face of multilevel MRI abnormalities. In the present series, 44 patients with MRI abnormalities of $2.7 \pm 1.1$ levels were successfully treated by anterior operation at a single (35) or two (9) optimal levels, which, as opposed to the procedures with several levels involved, has the advantage of less perioperative morbidity and greater postoperative stability with a smaller non-union rate.

\section{Issues concerning invasive electrode placements}

In the light of increasing emphasis on minimal invasiveness for any diagnostic test, a supplementary explanation should be added for the invasive aspects of our method. This intraoperative SCEP study, although providing a good quality measure that allows rapid feedback to the surgeons in only a few minutes, requires insertion of corkscrew-type needles into the scalp and a flexible electrode into the dorsal epidural space after preoperative general anesthesia. Immediately before the SCEP recording, placement of recording needle electrodes into either the intervertebral disc or the ligamentum flavum interrupts the surgical procedure for a few additional minutes. These invasive placements of spinal stimulating and recording electrodes, although causing no adverse effects in our series, carry a minimal risk, if any, of hemorrhagic, traumatic or infectious complications. The techniques, however, can be justified if applied for the purpose of monitoring to prevent iatrogenic complication, as described in recent reviews of modern intraoperative spinal cord monitoring $[10,11]$. In fact, both the scalp and the epidural stimulating electrodes and some of the recording electrodes used in the present study also served for combined motor and sensory tract monitoring during the subsequent decompression procedure. A low risk for accelerating disc degeneration by needle electrode insertion into the intervertebral disc relies on one previous study [15], which reported that the lumbar discs that had undergone diagnostic discography in 36 patients showed no additional disc abnormalities 10-20 years later compared with adjacent uninvestigated discs. Nevertheless, only with precautions for patient safety in conjunction with selective use of the test in proper clinical contexts, will the SCEP study maximize its impact as a diagnostic procedure.

\section{Conclusions}

Electrophysiological exploration revealed that the spinal sensory and motor pathways were functionally compromised at the same single level in 78 out of 83 DCM patients (94\%) and at two separate levels in the remaining 5 patients $(6 \%)$ in the presence of multilevel MRI abnormalities. The site of conduction block had a sufficient degree of cord compression shown by MRI with either the smallest $(81 \%)$ or the second smallest $(19 \%)$ anteroposterior diameter and cross-sectional area of the cord. A relatively older cohort of the present series of DCM patients showed a high incidence of conduction block at $\mathrm{C} 3-4$ or $\mathrm{C} 4-5$ in both A-SCEP (80\%) and D-SCEP studies (81\%). Combined A-SCEP and D-SCEP studies, which can be performed during surgery, before decompression procedures, serve as useful additions to MRI in precisely localizing the sites responsible for the main functional change, thereby directing the anterior surgical intervention to a single or two optimal levels in spite of multilevel MRI abnormalities.

\section{Data archiving}

All relevant data are within this manuscript and raw data are archived by the authors.

\section{Compliance with ethical standards}

Conflict of interest The authors declare that they have no competing interests. 


\section{References}

1. Nouri A, Tetreault L, Singh A, Karadimas SK, Fehlings MG. Degenerative cervical myelopathy: epidemiology, genetics, and pathogenesis. Spine. 2015;40:E675-93.

2. Kato F, Yukawa Y, Suda K, Yamagata M, Ueta T. Normal morphology, age-related changes and abnormal findings of the cervical spine. Part II: magnetic resonance imaging of over 1,200 asymptomatic subjects. Eur Spine J. 2012;21:1499-507.

3. Tani T, Ishida K, Ushida T, Yamamato H. Intraoperative electroneurography in the assessment of the level of operation for cervical spondylotic myelopathy in the elderly. J Bone Joint Surg. 2000;82:269-74.

4. Tani T, Ushida T, Taniguchi S, Kimura J. Age related shift in the primary sites of involvement in cervical spondylotic myelopathy from lower to upper levels. J Neurol Neurosurg Psychiatry. 2002;73:316-8.

5. Tadokoro N, Tani T, Ikeuchi M, Takemasa R, Kida K, Ikemoto T, et al Descending spinal cord evoked potentials in cervical spondylotic myelopathy: characteristic waveform changes seen at the lesion site. Clin Neurophysiol. 2014;125:202-7.

6. Japanese Orthopaedic Association. Scoring system (17-2) for cervical myelopathy. Nippon Seikeigeka Gakkai Zasshi. 1994;68:498

7. Kimura J. Electrodiagnosis in diseases of nerve and muscle: principles and practice. 4th ed. New York, USA: Oxford University Press; 2013. p. 49-73.
8. Ikemoto T, Tani T, Taniguchi S, Ikeuchi M, Kimura J. Effects of experimental focal compression on excitability of human median motor axons. Clin Neurophysiol. 2009;120:342-7.

9. Kimura J. Electrodiagnosis in diseases of nerve and muscle: principles and practice. 4th ed. New York, USA: Oxford University Press; 2013. p. 288-96.

10. Tani T, Saito T, Kawasaki M, Tadokoro N, Kimura J. Spinal cord monitoring for high risk spinal surgery. In: Johari A, Luk KDK, Waddell JP, editors. Current progress in orthopedics. Maharashtra, Mumbai: Three Life Media; 2016. p. 232-53. Vol. 2, Chapter 13.

11. McDonald DB, Skinner S, Shils J, Yingling C. Intraoperative motor evoked potentia monitoring-a position statement by the American Society of Neurophysiological Monitoring. Clin Neurophysiol. 2013;124:2291-316.

12. Goodridge AE, Feasby TE, Ebers GC, Brown WF, Rice GP. Hand wasting due to mid-cervical spinal cord compression. Can J Neurol Sci. 1987;14:309-11.

13. Kameyama T, Hashizume Y, Sobue G Morphologic features of the normal human cadaveric spinal cord. Spine. 1996;21:1285-90.

14. Al-Mefty O, Harkey LH, Middleton TH, Smith RR, Fox JL. Myelopathic cervical spondylotic lesions demonstrated by magnetic resonance imaging. J Neurosurg. 1988;68:217-22.

15. Flanagan MN, Chung BU. Roentgenographic changes in 188 patients 10-20 years after discography and chemonucleolysis. Spine. 1986;11:444-8. 